\title{
Optical Absorption and Electron Injection of 4-(Cyanomethyl)benzoic Acid Based Dyes: A DFT Study
}

\author{
Yuehua Zhang, ${ }^{1,2}$ Penghui Ren, ${ }^{1,2}$ Yuanzuo Li, ${ }^{2}$ Runzhou Su, ${ }^{2}$ and Meiyu Zhao ${ }^{3}$ \\ ${ }^{1}$ College of Science, Jiamusi University, Jiamusi, Heilongjiang 154007, China \\ ${ }^{2}$ College of Science, Northeast Forestry University, Harbin, Heilongjiang 150040, China \\ ${ }^{3}$ Institute of Theoretical Simulation Chemistry, Academy of Fundamental and Interdisciplinary Sciences, \\ Harbin Institute of Technology, Harbin 150080, China
}

Correspondence should be addressed to Yuanzuo Li; yuanzuo.li@gmail.com

Received 11 April 2015; Revised 18 June 2015; Accepted 25 June 2015

Academic Editor: Dino Villagrán

Copyright (C) 2015 Yuehua Zhang et al. This is an open access article distributed under the Creative Commons Attribution License, which permits unrestricted use, distribution, and reproduction in any medium, provided the original work is properly cited.

Density functional theory (DFT) and time-dependent density functional theory (TDDFT) calculations were carried out to study the ground state geometries, electronic structures, and absorption spectra of 4-(cyanomethyl)benzoic acid based dyes (AG1 and AG2) used for dye-sensitized solar cells (DSSCs). The excited states properties and the thermodynamical parameters of electron injection were studied. The results showed that (a) two dyes have uncoplanar structures along the donor unit and conjugated bridge space, (b) two sensitizers exhibited intense absorption in the UV-Vis region, and (c) the excited state oxidation potential was higher than the conduction band edge of $\mathrm{TiO}_{2}$ photoanode. As a result, a solar cell based on the 4-(cyanomethyl)benzoic acid based dyes exhibited well photovoltaic performance. Furthermore, nine dyes were designed on the basis of AG1 and AG2 to improve optical response and electron injection.

\section{Introduction}

Since O'Regan and Grätzel reported the Dye-sensitized solar cells (DSSCs) [1], DSSCs used as devices that convert visible light into electricity based on the photosensitization of wide band gap metal oxide semiconductors have attracted wide interest as a low cost of solar energy conversion. The widened absorption spectra range of dyes and the electron transfer capability from excited dyes to the semiconductor $\mathrm{TiO}_{2}$ were viewed as two important factors for conversion efficiency of DSSC [2-4]. Molecular design needs to meet the below requirements [2]: in order to absorb more photons, the dye must have broad absorption spectrum in the visible range; in order to separate charge efficiently, the excited state of the dyes must have a charge transfer ability to the electrode; in order to inject electrons into the electrode, the lowest energy level of unoccupied molecular orbital (LUMO) of the dye energy must be higher than that of the conduction band edge of the semiconductor $\mathrm{TiO}_{2}$; in order to obtain the electron from the electrolytic solution, the highest occupied molecular orbital (HOMO) energy must be lower than the reduction potential of the electrolytic solution $\mathrm{I}^{-} / \mathrm{I}_{3}{ }^{-}$oxide; for practical application of the battery, the dye molecules must have longterm stability; at the same time, it can withstand more than $10^{8}$ times of oxidation-reduction process.

Until now, the steady efficiency of the DSSCs is kept by a polypyridyl ruthenium sensitizer [2, 5-7]; however, we have to face the practical problems of ruthenium sensitizer utility, such as a very expensive cost. As an alternative, metal free organic sensitizers have received an increasing attention and can obtain efficiencies reaching 10\% [8-17]. In terms of metalfree DSSCs, donor- $\pi$-bridge-acceptor $(\mathrm{D}-\pi-\mathrm{A})$ is a typical structure. Various organic dyes, such as coumarin $[8,9]$, porphyrins $[7,10]$ phenylene [11], carbazole dyes [12, 13], indoline dyes [14, 15], and bis-dimethyl fluoroaniline (JKs) [16] and Ds series [17], have been synthesized. The D- $\pi$ A molecular structures are constructed by electron donor (D), $\pi$-conjugated linker and electron acceptor (A) moieties. Meanwhile, the modified donor part and $\pi$-conjugated bridge are main channel for molecular design of DSSCs. 
Recently, Gupta et al. [18] reported 4-(cyanomethyl)benzoic acid based dyes, which displayed promising properties in the development of photovoltaic devices [18]. Experiment demonstrated that the designed dyes, by means of 4-(cyanomethyl)benzoic acid as an attachment group and replacement of the bridging para-phenyl group in the triaryl donor unit with a thiophene group, displayed a power conversion efficiency of $6.37 \%$. For understanding the relationship between the molecular structure and performance, the molecular simulation was done. And the theoretical simulation approach is more efficient, whose results have more significance for the synthesis of new forwardlooking photosensitizer dyes. As a modeling method, quantum chemistry methods, especially density functional theory (DFT) and time-dependent DFT (TD-DFT), were commonly used for theoretical treatment of structures and electronic and absorption spectra of ruthenium sensitizer, metal-free sensitizer, natural dyes, and heterojunction organic solar cell [19-25] and for studying the optical materials with charge transfer character and the chemical mechanism of the surface enhancement Raman spectroscopy [26-28]. In current work, we performed a quantum chemistry calculation on the geometries, electronic structures, hyperpolarizabilities, and the Ultraviolet-Visible (UV-Vis) spectrum of 4-(cyanomethyl)benzoic acid based dyes (AG1 and AG2), and the solvent effect was considered. The absorption peaks and electron transfer were analyzed. Excited state oxidation potentials and thermodynamically driving force of the electron injection process were examined. In addition, nine dyes were designed on the basis of AG1 and AG2.

\section{Methods}

Geometric optimizations of two molecules were performed with density functional theory (DFT) [29], using B3LYP functional [30] at the $6-31 \mathrm{G}(\mathrm{D})$ basis set. Based on the optimized ground state geometry, vibration frequencies were done at the same level. There was no imaginary vibration frequency in the minimum energy structure in the potential energy landscape. The total static first hyperpolarizability can be written as follows [31]:

$$
\beta_{\mathrm{Tol}}=\sqrt{\beta_{X}^{2}+\beta_{y}^{2}+\beta_{Z}^{2}} .
$$

Individual static components in the above is calculated from

$$
\beta_{i}=\beta_{i i i}+\frac{1}{3} \sum_{i \neq j}\left(\beta_{i j j}+\beta_{j i j}+\beta_{j j i}\right),
$$

where $\beta_{i j k}(i, j, k=x, y, z)$ are tenser components of hyperpolarizability. Due to the Kleinman symmetry, we finally obtain the equation that has been employed:

$$
\begin{aligned}
& \beta_{\text {tot }}=\left[\left(\beta_{x x x}+\beta_{x y y}+\beta_{x z z}\right)^{2}+\left(\beta_{y y y}+\beta_{y z z}+\beta_{y x x}\right)^{2}\right. \\
& \left.+\left(\beta_{z z z}+\beta_{z x x}+\beta_{z y y}\right)^{2}\right]^{1 / 2} .
\end{aligned}
$$

The absorption spectra of them were obtained with timedependent DFT (TD-DFT) [32] by using the Cam-B3LYP functional [33] and the 6-31G(D) basis set. Chloroform solution in CPCM method was employed in calculations according to the experimental setting [18]. By comparing the bond lengths, the frontier molecular orbital energy levels, energy gaps, absorption spectra, and excited state oxidation potentials and thermodynamically driving force of the electron injection, one studies the stable structure, optical properties, and electron injection of dyes. All the calculations were done with the Gaussian 09 package [34].

\section{Results and Discussion}

3.1. Geometric Structures. The chemical structure of AG1 and AG2 was shown in Figure 1, where the serial numbers of atoms was also indicated. Two dyes are typical D$\pi$-A structures, with the same terminal accepting group (4-(Cyanomethyl)benzoic acid moiety) and different $\pi$ conjugated spacers (the paraphenyl unit for AG1 and a thiophene group for AG2) and different donor units (triarylamine group for AG1 and triaryl donor for AG2). The AG1 and AG2 ground state geometries were optimized by 6-31G (d), and selected structural parameters were listed in Table 1. As shown in Table 1, for AG1, there is a twisted structure upon donor unit, where dihedral angle of C1-C2-N3-C4 and C2-N3-C4-C5 is -45.45954 degrees and -34.89785 degrees, respectively, and mean value of two twisted dihedral angles is 40 degrees. For AG2, C1-C2-N3-C4 and C2-N3-C4-S5 also display a twisted structure (dihedral angle of C1-C2N3-C4 and C2-N3-C4-S5 is about -45.89085 and -39.89847 , resp.), and mean value is 43 degrees. Recent report indicated that some higher performance dyes (such as Ds and bisdimethylfluorenylamino dyes $[16,17]$ ) have different forms of the twisted configuration on donor unit, and this twisted configuration is helpful to suppress the dyes aggregation on the surface of semiconductor. Different to the other dyes, AG1 and AG2 have uncoplanar structure upon the bridge moiety. For AG1, the dihedral angles of D (C6-C7C8-S9), D (S9-C10-C11-S12), and D (C14-C15-C16-C17) are 21.07776, -17.10793 , and 162.15200 for thiophen rings and benzene rings, respectively. But for the AG2, the twisted degree of thiophene rings of conjugated bridge is lower than that of AG1; for example, D (S5-C6-C7-S8) and D (S8-C9C10-S11) are -14.84480 and 14.78207 , respectively. While for ethylbenzene unit and acceptor units, there is a well coplanar configuration for two dyes.

3.2. Molecular Orbitals. The frontier molecular orbital energies of the dyes AG1 and AG2 were listed in Table 2, and the plot surface of molecular orbitals was shown in Figure 2. For AGl, the highest occupied MO (HOMO) is $-4.96 \mathrm{eV}$. From electron density of molecular orbitals in Figure 2, electron density of HOMO is delocalized throughout the dye, which belongs to the dye $\pi$ orbital. The lowest unoccupied MO (LUMO), $-2.51 \mathrm{eV}$ above the HOMO, is an $\pi^{*}$ orbital delocalized, and electron density of LUMO is located on the conjugated bridge and cyanoacrylic acid moiety. So if electron transitions from HOMO to LUMO, excitation should result in electron transfer from triarylamine group to 4-(cyanomethyl)benzoic acid moiety. Energy levels of 


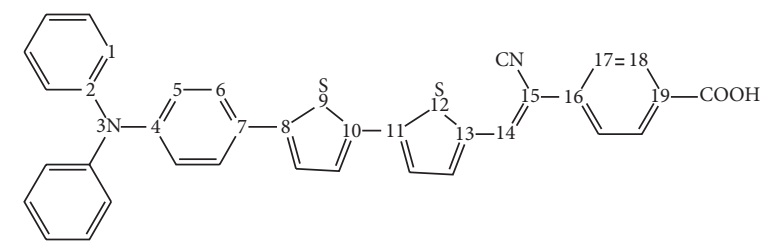

AG1

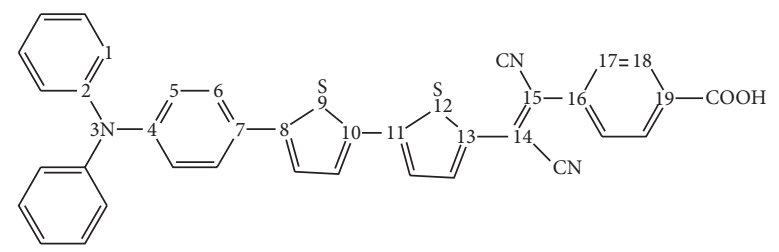

AG1-a

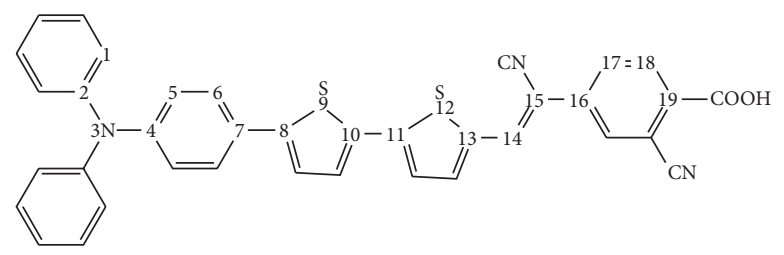

AG1-b

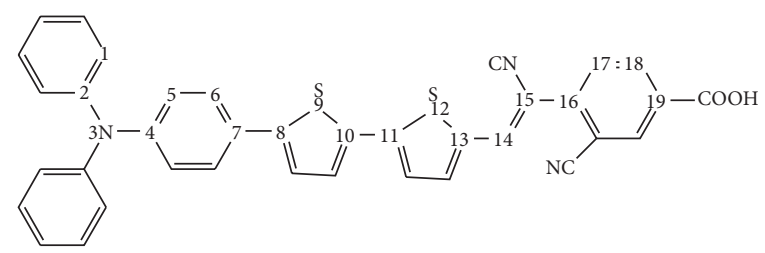

AG1-c

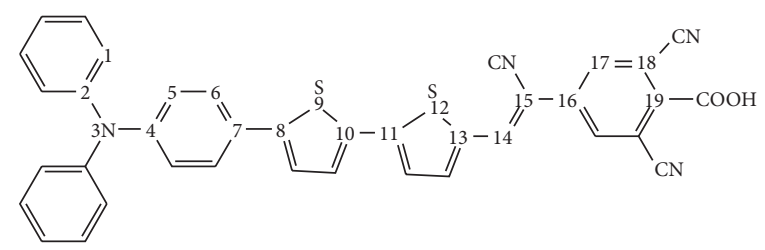

AG1-d

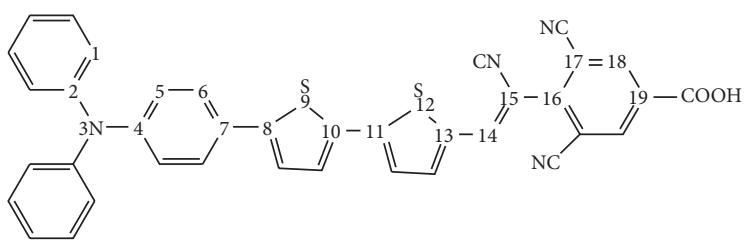

AG1-e

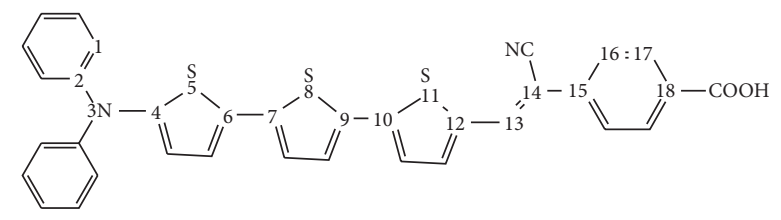

AG2

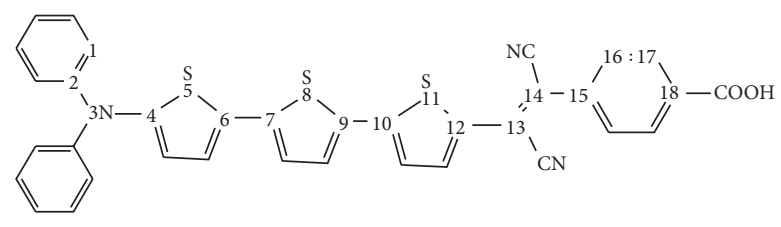

AG2-a

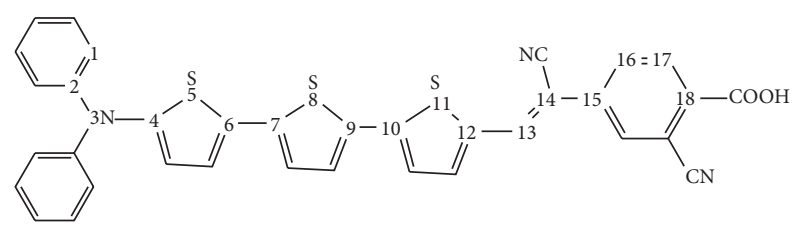

AG2-b

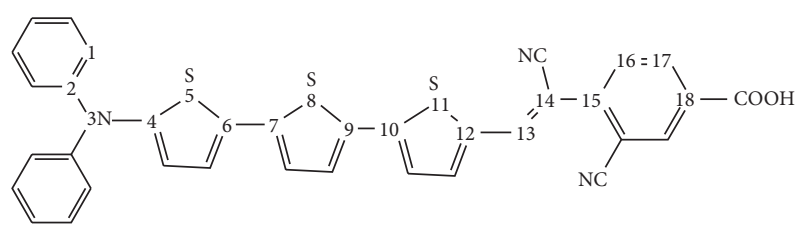

AG2-c

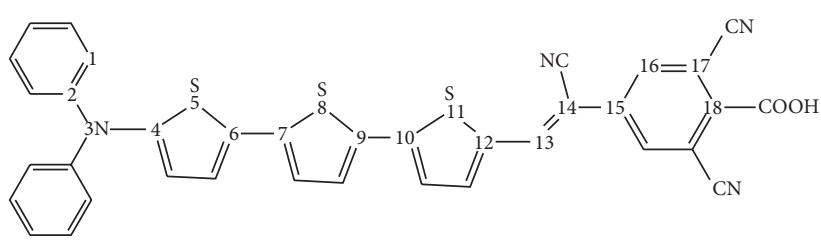

AG2-d

FIGURE 1: Chemical structures of two molecules AG1 and AG2 and designed nine molecules (AG1, AG1-a, AG1-b, AG1-c, AG1-d, AG1-e, AG2, AG2-a, AG2-b, G2-c, and AG2-d).

other molecular orbitals (such as $\mathrm{HOMO}-1, \mathrm{HOMO}-2$, and LUMO+1) were listed in supporting materials (see Table S1 in Supplementary Material available online at http://dx.doi.org/ $10.1155 / 2015 / 402746)$. From Table S1, it was found that the LUMO +1 is $-1.47 \mathrm{eV}$ above the LUMO and is localized within the conjugated bridge and cyanoacrylic acid moiety (where more electrons are located in benzene ring of conjugated bridge compared with LUMO). Similar electron density was found for LUMO +2 , lying at $-0.92 \mathrm{eV}$. But for LUMO lying at $-0.55 \mathrm{eV}$, electron density is localized in triarylamine group, 
AG1
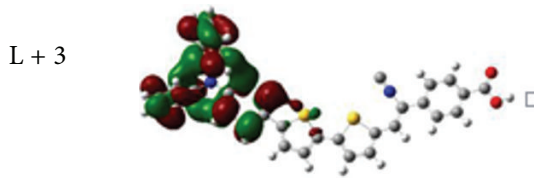

$\mathrm{L}+2$

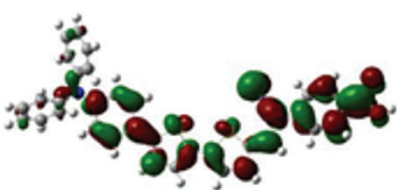

$\mathrm{L}+1$

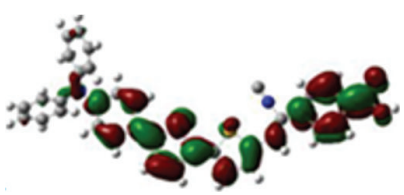

L

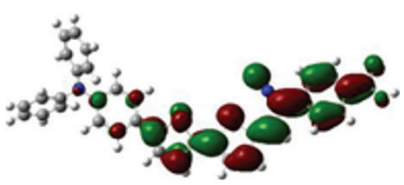

$\mathrm{H}$

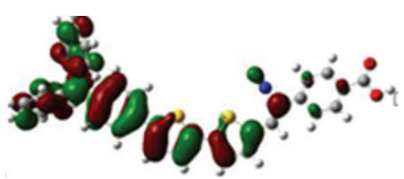

$\mathrm{H}-1$
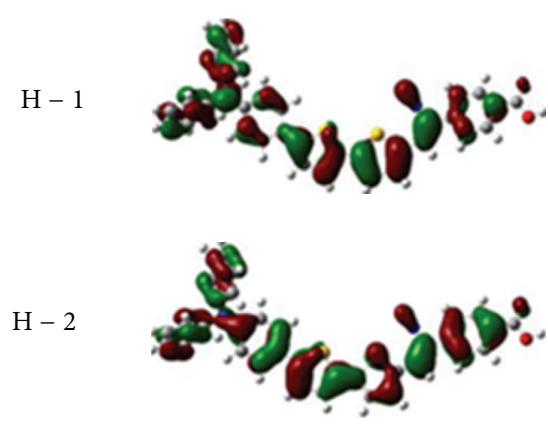

$\mathrm{H}-3$

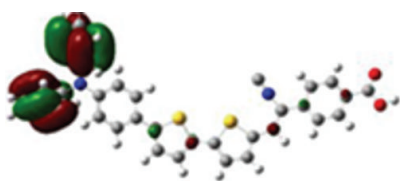

AG2
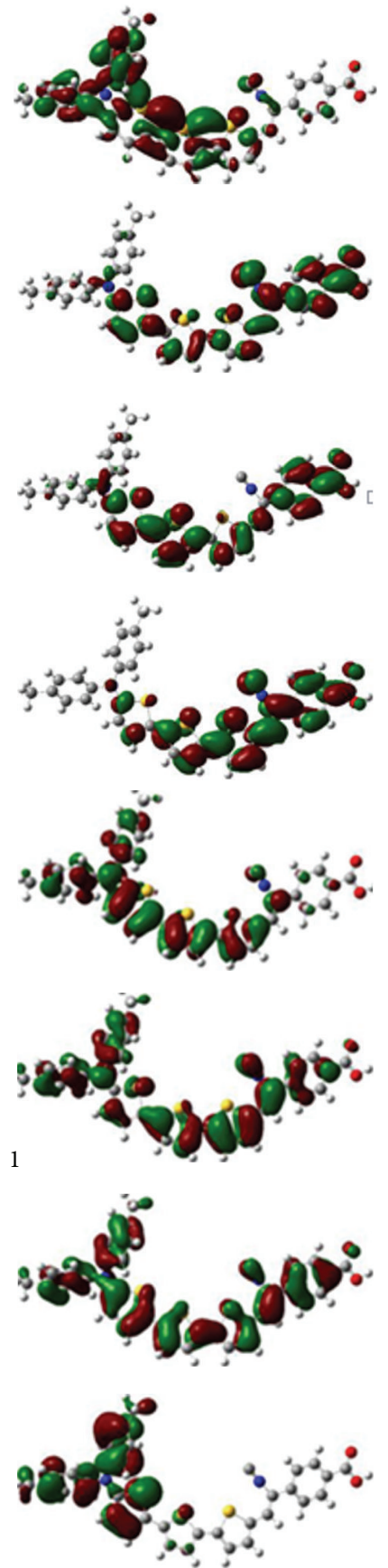

FIGURE 2: The plot surface of molecular orbitals of two compounds (AG1 and AG2).

which is similar to the electron density of HOMO-3 lying at $-6.87 \mathrm{eV}$.

For AG2, Table 2 shows that the energy level of HOMO is at $-4.77 \mathrm{eV}$, which has a difference of $0.19 \mathrm{eV}$ compared with HOMO of AG1. The energy level of LUMO is $-2.52 \mathrm{eV}$, which is nearly changed compared with AG1. So the structure modification from AG1 to AG2 can only change the energy level of HOMO. The HOMO is $\pi$ orbital delocalized throughout the dye, and electron density of LUMO with character of $\pi^{*}$ orbital is located on the conjugated bridge and 4-(cyanomethyl)benzoic acid moiety, which means that electron transition from HOMO to LUMO should result in electron transfer from triarylamine group to 4(cyanomethyl)benzoic acid moiety. Energy levels of LUMO+1 and $\mathrm{LUMO}+2$ for AG2 are lower than those of AG1, and $\mathrm{HOMO}+1$ and $\mathrm{HOMO}+2$ of $\mathrm{AG} 2$ are higher than those 
TABLE 1: Calculated bond lengths and dihedral angle for two compounds, respectively.

\begin{tabular}{lccc}
\hline \multicolumn{1}{c}{ AG1 } & \multicolumn{2}{c}{ AG2 } \\
\hline C1-C2-N3-C4 & -45.45954 & C1-C2-N3-C4 & -45.89085 \\
C2-N3-C4-C5 & -34.89785 & C2-N3-C4-S5 & -39.89847 \\
C6-C7-C8-S9 & 21.07776 & S5-C6-C7-S8 & -14.84480 \\
S9-C10-C11-S12 & -17.10793 & S8-C9-C10-S11 & 14.78207 \\
S12-C13-C14-C15 & -0.83014 & C11-C12-C13-C14 & 1.10018 \\
C13-C14-C15-C16 & 179.55183 & C12-C13-C14-C15 & -179.35721 \\
C14-C15-C16-C17 & 162.15200 & C14-C15-C16-C17 & 179.11762 \\
C15-C16-C17-C18 & -179.05968 & C15-C16-C17-C18 & 0.35682 \\
C17-C18-C19-C20 & -179.93959 & C16-C17-C18-C19 & 179.90957 \\
C2-N3 & 1.42566 & C2-N3 & 1.42882 \\
N3-C4 & 1.41163 & N3-C4 & 1.38802 \\
C7-C8 & 1.46110 & C6-C7 & 1.44238 \\
C10-C11 & 1.44308 & C9-C10 & 1.44052 \\
C13-C14 & 1.42967 & C12-C13 & 1.42861 \\
C14-C15 & 1.36605 & C13-C14 & 1.36669 \\
C15-C16 & 1.47756 & C14-C15 & 1.47704 \\
C19-C20 & 1.48352 & C18-C19 & 1.48327 \\
\hline
\end{tabular}

TABLE 2: Energy levels of HOMO and LUMO and energy gap (eV).

\begin{tabular}{lcc}
\hline & AG1 & AG2 \\
\hline $\mathrm{H}$ & -4.96 & -4.77 \\
$\mathrm{~L}$ & -2.51 & -2.52 \\
Gap & -2.45 & -2.25 \\
\hline
\end{tabular}

corresponding energy levels of AG1. The energy gaps (H-L gap) of AG1 and AG2 are $-2.45 \mathrm{eV}$ and $-2.25 \mathrm{eV}$, respectively, and $\mathrm{H}-\mathrm{L}$ gap of $\mathrm{AG} 2$ is reduced with respect to that of AG1.

3.3. Absorption. The transition energy and oscillator strength were listed in Table 3, and the first three excitations were included in current calculation. One also checked the basis set effects with 6-31G (d), 6-31G (d,p), 6-311G (d), and $6-311+G(d, p)$ basis sets. Transition energy and oscillator strength for the first excited state (S1) of AG1 are calculated to be $452.64 \mathrm{~nm}(f=1.79)$ with Cam-B3LYP/6-31G $(\mathrm{d})$ and 452.87 ( $f=1.7863$ ) with Cam-B3LYP/6-31G $(\mathrm{d}, \mathrm{p})$, and hence there is no scarce change upon the addition of one polarized functional. Similar trend was found for the two basis sets 6 $311+\mathrm{G}(\mathrm{d})$ and $6-311+\mathrm{G}(\mathrm{d}, \mathrm{p})$; that is, $\mathrm{S} 1$ state is 465.08 (1.7598) and 465.60 (1.7556), respectively. Compared with the general basis set $6-31 \mathrm{G}(\mathrm{d})$, there is redshift about $6 \mathrm{~nm}$ by using basis set $6-311 \mathrm{G}(\mathrm{d})$; at the same time, upon the addition of diffuse functional, it is 465.08 ( $f=1.7598)$ with the basis set 6$311+\mathrm{G}(\mathrm{d})$, and it makes redshift about $7 \mathrm{~nm}$ compared with 6-311G(d). Therefore, evaluation of transition energy of AG1 can be affected by the increase basis set and addition of diffuse functional. For AG2, transition energy of $S 1$ is $487.83 \mathrm{~nm}(f=$ $1.6155)$ with 6-31G $(\mathrm{d})$ and $488.09(f=1.6129)$ with $6-31 \mathrm{G}$ $(d, p)$, respectively, and the change is very small. Upon using the 6-311G (d), transition energy of AG2 is $494 \mathrm{~nm}(495 \mathrm{~nm}$ for experiment [18]). One also checked the bases $6-311+G(d)$ and $6-311+G(d, p)$; unfortunately, the two basis sets are not convergent. Therefore, the basis set 6-311G (d) will be used in the next designed molecules and discussion.

It is seen in Table 3 that the dominant absorption band is found to be the first excited state (S1 state) in the UVVis spectra region. When paying attention to the difference of molecule absorption peaks, one can find that there is difference about $36 \mathrm{~nm}$ between AG1 and AG2.

For AG1, the absorption peak is the first excited state ( $\mathrm{S} 1$ state), and oscillator strength is 1.7797 , which is the largest strength among the three states. Other states $(f>0.1)$ are S2 state. Electron transition information was listed in Table S2. From Table S2, the first excited state showed a $\mathrm{HOMO} \rightarrow$ LUMO transition (transition possibility is $58.8 \%$; see Table S2), and electron density change (from HOMO to LUMO, in Figure 2) shows this state is intramolecular charge transfer state (ICT); for more high excited state (S2), its absorption strength is lower than that of S1, which displays a HOMO-1 $\rightarrow$ LUMO transition with the weight (47.1\%). From HOMO-1, electron density is delocalized over the whole molecule, and LUMO is located on the bridge and acceptor units; so this state is ICT state. Due to the similar distribution of electron density between LUMO and $\mathrm{LUMO}+1$, the third state, in which electron transition is from $\mathrm{HOMO}$ to LUMO+1, is an ICT state.

Table S2 shows that the absorption peak of AG2 is the first excited state ( $\mathrm{S} 1$ state), and oscillator strength is 1.5986 , which is lower than that of AG1. Though AG2 has redshifted absorption compared with AG1, oscillator strength is lower for AG1. Other states $(f>0.1)$ are S2 state. For S1, this state showed a HOMO $\rightarrow$ LUMO transition. At the second excitation state (S2), the oscillator strength is 0.5939 , with the electron transition from HOMO-1 to LUMO transition; for $\mathrm{S} 3$, it includes $\mathrm{HOMO} \rightarrow \mathrm{LUMO}+1$ transition.

For DSSCs, electron transfer from donor to acceptor characterized by ICT state is beneficial for the electron aggregation on acceptor moiety and enhances the electron coupling effect between acceptor and semiconduction, because the first hyperpolarizabilities $(\beta)$ are directly proportional to the difference in the dipole moment between the HOMO and the LUMO orbitals $\left(\Delta \mu_{\mathrm{eg}}\right)$ and the transition dipole moment (oscillator strength) $\left(\mu_{\text {eg }}\right)$ and they are inversely proportional to the transition energy. The first hyperpolarizabilities can be written as follows $[35,36]$ :

$$
\beta \infty \frac{\Delta \mu_{\mathrm{eg}}\left(\mu_{\mathrm{eg}}\right)^{2}}{E_{\mathrm{eg}}^{2}},
$$

where $\Delta \mu_{\text {eg }}$ and $\mu_{\text {eg }}$ are difference in the dipole moment for ground state and excited state and the transition dipole and $E_{\text {eg }}$ is transition energy. After the geometry optimization, the first hyperpolarizabilities were calculated, which was listed in Table 4 . It can be seen that $\beta_{x x x}$ (along coordinate axis of molecular body) is a negative value that suggests the obvious negative charge distribution away from centralisation of the molecular nuclear charge. And the $\beta_{x x x}$ value of AG2 is larger than that of AG1. From Table 3, oscillator strength of AG1 is larger than that of AG2, and proportional value of 


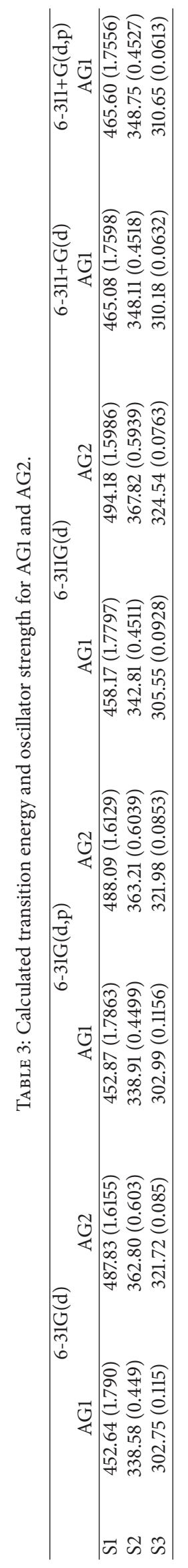


TABLE 4: Hyperpolarizability of AG1 and AG2.

\begin{tabular}{cccccccccccc}
\hline & $\beta_{x x x}$ & $\beta_{x x y}$ & $\beta_{x y y}$ & $\beta_{y y y}$ & $\beta_{x x z}$ & $\beta_{x y z}$ & $\beta_{y y z}$ & $\beta_{x z z}$ & $\beta_{y z z}$ & $\beta_{z z z}$ & $\beta_{\text {tot }}$ \\
\hline AG1 & -116132 & 1917 & -4563 & 1607 & 1704 & -962 & -164 & 162 & 71 & 14 & 120596 \\
AG2 & -145255 & 5296 & -8205 & 1315 & 5869 & -2688 & -191 & -221 & 268 & 197 & 153948 \\
\hline
\end{tabular}

TABLE 5: Calculated transition energy and oscillator strength designed dyes (AG1-X and AG2-X) in solvent phase.

\begin{tabular}{|c|c|c|c|c|c|}
\hline Dye & State & Contribution MO & $E(\mathrm{eV})$ & $\begin{array}{c}\text { Absorption peak } \\
\lambda(\mathrm{nm})\end{array}$ & $\begin{array}{c}\text { Strength } \\
f\end{array}$ \\
\hline AG1-a & 1 & $(57.0 \%) \mathrm{H} \rightarrow \mathrm{L}$ & 2.5768 & 481.15 & 1.4565 \\
\hline AG1-b & 1 & $(57.4 \%) \mathrm{H} \rightarrow \mathrm{L}$ & 2.6065 & 475.67 & 1.8080 \\
\hline AG1-c & 1 & $(57.4 \%) \mathrm{H} \rightarrow \mathrm{L}$ & 2.6158 & 473.98 & 1.7177 \\
\hline AG1-d & 1 & $(56.2 \%) \mathrm{H} \rightarrow \mathrm{L}$ & 2.4911 & 497.71 & 1.8109 \\
\hline AG1-e & 1 & $(55.5 \%) \mathrm{H} \rightarrow \mathrm{L}$ & 2.5975 & 477.32 & 1.6009 \\
\hline AG2-a & 1 & $(60.3 \%) \mathrm{H} \rightarrow \mathrm{L}$ & 2.3512 & 527.32 & 1.3658 \\
\hline AG2-b & 1 & $(60.2 \%) \mathrm{H} \rightarrow \mathrm{L}$ & 2.3847 & 519.92 & 1.6444 \\
\hline AG2-c & 1 & $(60.2 \%) \mathrm{H} \rightarrow \mathrm{L}$ & 2.4011 & 516.37 & 1.5839 \\
\hline AG2-d & 1 & $(59.4 \%) \mathrm{H} \rightarrow \mathrm{L}$ & 2.2535 & 550.18 & 1.6711 \\
\hline
\end{tabular}

transition energy between AG1 and AG2 is calculated to be 1.113. However, the value of the first hyperpolarizabilities for Ag2 is larger than that of AG1, which means that there is an obvious difference in the dipole moment (enhancing ICT character).

3.4. Driving Force of Electron Injection. Not only do the highperformance dyes used for DSSCs have well optical response in Ultraviolet-Visible spectral region, but also the excited electron should quickly be injected from the discontinuous energy level of dyes into the $\mathrm{CB}$ of the semiconductor titanium dioxide. Thermodynamically, driving force of the electron injection process can be described as $E_{\mathrm{TDF}}=E_{\mathrm{ex}}^{\mathrm{ox}}-$ $E_{\mathrm{cb}}$, where $E_{\mathrm{ex}}^{\mathrm{ox}}$ and $E_{\mathrm{cb}}$ stand for the excited state oxidation potential and $\mathrm{CB}$ edge, respectively. According to the Rehm and Weller equation $[37,38], E_{\mathrm{ex}}^{\mathrm{ox}}$ can be calculated as follows:

$$
E_{\mathrm{ex}}^{\mathrm{ox}}=E_{\mathrm{gr}}^{\mathrm{ox}}-E_{00} \text {, }
$$

where $E_{\mathrm{ex}}^{\mathrm{ox}}$ and $E_{\mathrm{gr}}^{\mathrm{ox}}$ are the excited and ground state oxidation potentials, $E_{00}$ is the electronic transition energy, and the ground state oxidation potential $E_{\mathrm{gr}}^{\mathrm{ox}}$ is computed from the HOMO energy. The value of $E_{\mathrm{ex}}^{\mathrm{ox}}$ is calculated to be $-1.509 \mathrm{~V}$ (for AG1) and $-1.357 \mathrm{~V}$ (for AG2), which is more negative than the $\mathrm{CB}$ edge of $\mathrm{TiO}_{2}(0.5 \mathrm{~V}$ versus normal hydrogen electrode (NHE)) [39], and electron injection of AG1 is more easy than that of AG2 owing to the bigger difference between the excited state oxidation potentials and CB edge.

3.5. Molecular Design. The designed molecule structures with the addition of pull election group (-CN) were shown in Figure 1, and those dyes were named after AG1-a, AG1b, AG1-c, AG1-d, AG1-e, AG2-a, AG2-b, AG2-c, and AG2d. Calculated transition energies and absorption strengths of absorption peak were listed in Table 5. Table 5 shows that maxima of absorption peaks are $481.15 \mathrm{~nm}$ for AG1a, $475.67 \mathrm{~nm}$ for AG1-b, $473.98 \mathrm{~nm}$ for AG1-c, $497.71 \mathrm{~nm}$ for AG1-d, and $477.32 \mathrm{~nm}$ for AG1-e, which are redshifted compared with the original molecule AG1 $(452 \mathrm{~nm})$. The states are all first excited state for the five dyes and electron transition corresponding to the $\mathrm{HOMO} \rightarrow$ LUMO transition. Among the designed AG1-X $(X=a, \ldots, e)$, the oscillator strengths of AG1-b and AG1-d are larger than that of AG1, meaning that the absorption ability has been enhanced. For designed AG2-X, the values of $\lambda_{\max }$ are $527.32 \mathrm{~nm}$ for AG2a, $519.92 \mathrm{~nm}$ for AG2-b, $516.37 \mathrm{~nm}$ for AG2-c, and $550.18 \mathrm{~nm}$ for AG2-d. And the absorption strengths are in this order AG2-a $<$ AG2-c < AG2-b < AG2-d. Among the designed dyes AG2-X (X = a, .., d), AG2-d has the largest absorption peak and strength. Table 6 shows the first hyperpolarizability of designed dyes (AG1-X and AG2-X) in solvent phase, and for AG1-X, the first hyperpolarizability is in this order AG1 < AG1-c < AG1-a < AG1-b < AG1-e < AG1-d; for AG2-X, the first hyperpolarizability is AG2 < AG2-c $<$ AG2-b $<$ AG2-a $<$ AG2-d. Therefore, the introduction of group $(-\mathrm{CN})$ not only results in the redshifted absorption but also enhances the first hyperpolarizability (Table 6).

To evaluate driving force of electron injection, the energy levels of the excited states of the designed dyes were calculated which are listed in supporting materials in Table S3, and the corresponding energy level of conduction bands of $\mathrm{TiO}_{2}$, ground state, and singlet excited states of designed dyes (AG1$\mathrm{X}$ and AG2-X) were shown in Figure 3. As shown, for AG1$\mathrm{X}$, the values of $E_{\mathrm{ex}}^{\mathrm{ox}}$ of them are all more negative than that of the $\mathrm{CB}$ edge of $\mathrm{TiO}_{2}(0.5 \mathrm{~V}$ versus normal hydrogen electrode (NHE)); so the electron injection for designed dyes is thermodynamically allowed. Among the five designed molecules, AG1-d has the largest absorption wavelength and absorption strength, and thus it could utilize more sunlight energy, which can be considered as a candidate for the use in the field of DSSCs. For AG2-X, the four designed dyes display the high excited state oxidation potentials above the CB edge of $\mathrm{TiO}_{2}$, and thus electron injection for the four dyes AG2-X 


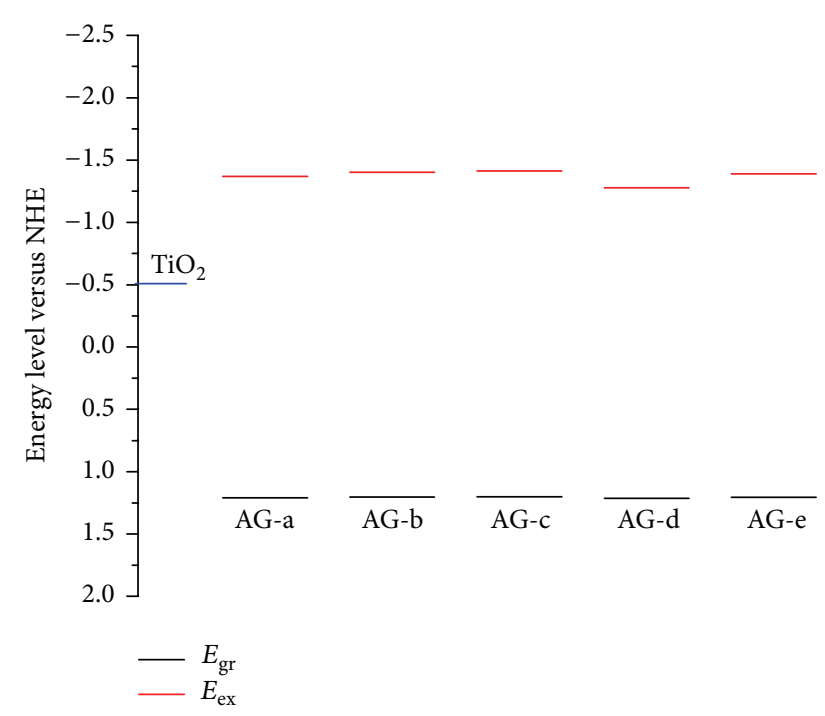

(a)

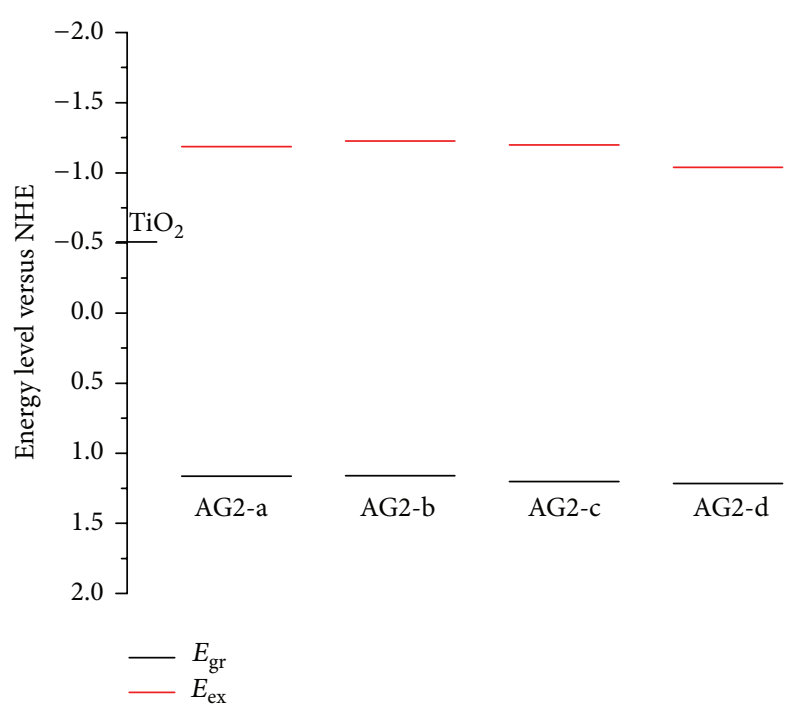

(b)

FIGURE 3: Energy levels of conduction bands of $\mathrm{TiO}_{2}$, ground state, and singlet excited states of designed dyes AG1-X (a) and AG2-X (b).

TABLE 6: Hyperpolarizability of designed dyes (AG1-X and AG2-X) in solvent phase.

\begin{tabular}{ccccccc}
\hline & AG1 & AG1-a & AG1-b & AG1-c & AG1-d & AG1-e \\
\hline$\beta_{\text {tot }}$ & 120596 & 183068 & 183681 & 169744 & 278726 & 194016 \\
\hline & AG2 & AG2-a & AG2-b & AG2-c & AG2-d & \\
\hline$\beta_{\text {tot }}$ & 153948 & 245671 & 240689 & 223810 & 374611 & \\
\hline
\end{tabular}

can occur. The value of $E_{\mathrm{ex}}^{\mathrm{ox}}$ is in this order AG2-d $<$ AG2-a $<$ AG2-c $<$ AG2-b.

\section{Conclusion}

The ground state geometries, electronic structures, and the first hyperpolarizability of dyes (AG1 and AG2) were investigated by means of DFT calculations with hybrid functional B3LYP, and the UV-Visible spectra were studied by TD-DFT methods with cam-B3LYP functional, and different basis sets were used in this work. Optimized results showed that AG1 and AG2 display the twisted structures. The HOMO-LUMO electronic transition describes their lowest singlet excited state. Absorption peak of AG2 is redshifted compared with AG1, meaning that AG2 can absorb more solar energy. The excited oxidation potentials of AG1 and AG2 are both higher than $\mathrm{CB}$ edge of the semiconductor titanium dioxide, suggesting that electron injection can occur thermodynamically. Therefore, the improvement of absorption efficiency for AG2 results in the high performance. Moreover, one designed nine dyes based on AG1 and AG2 by introducing the group (-CN). The results show that the introduction of group $(-\mathrm{CN})$ not only results in the redshifted absorption and the occurrence of the thermodynamically allowed electron injection but also enhances the first hyperpolarizability.

\section{Conflict of Interests}

The authors declare no conflict of interests.

\section{Acknowledgments}

This work was supported by the Fundamental Research Funds for the Heilongjiang Provincial Youth Science Foundation (Grant no. QC2013C006) and the National Natural Science Foundation of China (Grant nos. 11404055 and 11374353).

\section{References}

[1] B. O’Regan and M. Grätzel, "A low-cost, high-efficiency solar cell based on dye-sensitized colloidal $\mathrm{TiO}_{2}$ films," Nature, vol. 353, no. 6346, pp. 737-740, 1991.

[2] A. Hagfeldt, G. Boschloo, L. C. Sun, L. Kloo, and H. Pettersson, "Dye-sensitized solar cells," Chemical Reviews, vol. 110, no. 11, pp. 6595-6663, 2010.

[3] R. K. Kanaparthi, J. Kandhadi, and L. Giribabu, "Metal-free organic dyes for dye-sensitized solar cells: recent advances," Tetrahedron, vol. 68, no. 40, pp. 8383-8393, 2012.

[4] A. Mishra, M. K. R. Fischer, and P. Bäuerle, "Metal-Free organic dyes for dye-sensitized solar cells: from structure: property relationships to design rules," Angewandte Chemie International Edition, vol. 48, no. 14, pp. 2474-2499, 2009.

[5] M. Grätzel, "Recent advances in sensitized mesoscopic solar cells," Accounts of Chemical Research, vol. 42, no. 11, pp. 17881798, 2009.

[6] Y. Cao, Y. Bai, Q. Yu et al., "Dye-sensitized solar cells with a high absorptivity ruthenium sensitizer featuring a 2(hexylthio)thiophene conjugated bipyridine," The Journal of Physical Chemistry C, vol. 113, no. 15, pp. 6290-6297, 2009.

[7] L.-L. Li and E. W.-G. Diau, "Porphyrin-sensitized solar cells," Chemical Society Reviews, vol. 42, no. 1, pp. 291-304, 2013. 
[8] Z.-S. Wang, Y. Cui, K. Hara, Y. Dan-Oh, C. Kasada, and A. Shinpo, "A high-light-harvesting-efficiency coumarin dye for stable dye-sensitized solar cells," Advanced Materials, vol. 19, no. 8, pp. 1138-1141, 2007.

[9] Z.-S. Wang, Y. Cui, Y. Dan-Oh, C. Kasada, A. Shinpo, and K. Hara, "Molecular design of coumarin dyes for stable and efficient organic dye-sensitized solar cells," Journal of Physical Chemistry C, vol. 112, no. 43, pp. 17011-17017, 2008.

[10] M. Urbani, M. Grätzel, M. K. Nazeeruddin, and T. Torres, "Meso-substituted porphyrins for dye-sensitized solar cells," Chemical Reviews, vol. 114, no. 24, pp. 12330-12396, 2014.

[11] S. Mathew, H. Iijima, Y. Toude et al., "Optical, electrochemical, and photovoltaic effects of an electron-withdrawing tetrafluorophenylene bridge in a push-pull porphyrin sensitizer used for dye-sensitized solar cells," The Journal of Physical Chemistry C, vol. 115, no. 29, pp. 14415-14424, 2011.

[12] S. Kajiyama, Y. Uemura, H. Miura, K. Hara, and N. Koumura, "Organic dyes with oligo-n-hexylthiophene for dye-sensitized solar cells: relation between chemical structure of donor and photovoltaic performance," Dyes and Pigments, vol. 92, no. 3, pp. 1250-1256, 2012.

[13] C. Zafer, B. Gultekin, C. Ozsoy, C. Tozlu, B. Aydin, and S. Icli, "Carbazole-based organic dye sensitizers for efficient molecular photovoltaics," Solar Energy Materials and Solar Cells, vol. 94, no. 4, pp. 655-661, 2010.

[14] T. Horiuchi, H. Miura, K. Sumioka, and S. Uchida, "High efficiency of dye-sensitized solar cells based on metal-free indoline dyes," Journal of the American Chemical Society, vol. 126, no. 39, pp. 12218-12219, 2004.

[15] S. Ito, H. Miura, S. Uchida et al., "High-conversion-efficiency organic dye-sensitized solar cells with a novel indoline dye," Chemical Communications, no. 41, pp. 5194-5196, 2008.

[16] S. Kim, J. K. Lee, S. O. Kang et al., "Molecular engineering of organic sensitizers for solar cell applications," Journal of the American Chemical Society, vol. 128, no. 51, pp. 16701-16707, 2006.

[17] D. P. Hagberg, J.-H. Yum, H. Lee et al., "Molecular engineering of organic sensitizers for dye-sensitized solar cell applications," Journal of the American Chemical Society, vol. 130, no. 19, pp. 6259-6266, 2008.

[18] A. Gupta, V. Armel, W. Xiang, A. Bilic, and R. A. Evans, "New organic sensitizers using 4-(cyanomethyl)benzoic acid as an acceptor group for dye-sensitized solar cell applications," Dyes and Pigments, vol. 113, pp. 280-288, 2014.

[19] M. K. Nazeeruddin, F. De Angelis, S. Fantacci et al., "Combined experimental and DFT-TDDFT computational study of photoelectrochemical cell ruthenium sensitizers," Journal of the American Chemical Society, vol. 127, no. 48, pp. 16835-16847, 2005.

[20] N. Santhanamoorthi, C.-M. Lo, and J.-C. Jiang, "Molecular design of porphyrins for dye-sensitized solar cells: a DFT/ TDDFT study," Journal of Physical Chemistry Letters, vol. 4, no. 3, pp. 524-530, 2013.

[21] C.-R. Zhang, L. Liu, Z.-J. Liu et al., "Electronic structures and optical properties of organic dye sensitizer NKX derivatives for solar cells: a theoretical approach," Journal of Molecular Graphics and Modelling, vol. 38, pp. 419-429, 2012.

[22] H. X. Li and M. D. Chen, "Structure-property relationships for three indoline dyes used in dye-sensitized solar cells: TDDFT study of visible absorption and photoinduced charge-transfer processes," Journal of Molecular Modeling, vol. 19, no. 12, pp. 5317-5325, 2013.
[23] M. Snehalatha, C. Ravikumar, I. Hubert Joe, N. Sekar, and V. S. Jayakumar, "Spectroscopic analysis and DFT calculations of a food additive Carmoisine," Spectrochimica Acta Part A: Molecular and Biomolecular Spectroscopy, vol. 72, no. 3, pp. 654$662,2009$.

[24] Y. Z. Li, D. W. Qi, P. Song, and F. C. Ma, "Fullerene-based photoactive layers for heterojunction solar cells: structure, absorption spectra and charge transfer process," Materials, vol. 8, no. 1, pp. 42-56, 2015.

[25] J. Wang, H. Li, N.-N. Ma, L.-K. Yan, and Z.-M. Su, "Theoretical studies on organoimido-substituted hexamolybdates dyes for dye-sensitized solar cells (DSSC)," Dyes and Pigments, vol. 99, no. 2, pp. 440-446, 2013.

[26] M. Sun and H. Xu, "Direct visualization of the chemical mechanism in SERRS of 4-aminothiophenol/metal complexes and metal/4-aminothiophenol/metal junctions," ChemPhysChem, vol. 10, no. 2, pp. 392-399, 2009.

[27] M. T. Sun, Z. L. Zhang, L. Chen, S. X. Sheng, and H. X. $\mathrm{Xu}$, "Plasmonic gradient effects on high vacuum tip-enhanced raman spectroscopy," Advanced Optical Materials, vol. 2, no. 1, pp. 74-80, 2014.

[28] Y. Huang, Y. Fang, Z. Zhang, L. Zhu, and M. Sun, "Nanowiresupported plasmonic waveguide for remote excitation of surface-enhanced Raman scattering," Light: Science and Applications, vol. 3, article e199, 2014.

[29] J. M. R. Dreizler and E. K. U. Gross, Density Functional Theory, Springer, Heidelberg, Germany, 1990.

[30] A. D. Becke, "Density-functional thermochemistry. III. The role of exact exchange," The Journal of Chemical Physics, vol. 98, no. 7, pp. 5648-5652, 1993.

[31] D. A. Kleinman, "Nonlinear dielectric polarization in optical media," Physical Review, vol. 126, no. 6, pp. 1977-1979, 1962.

[32] R. E. Stratmann, G. E. Scuseria, and M. J. Frisch, "An efficient implementation of time-dependent density-functional theory for the calculation of excitation energies of large molecules," Journal of Chemical Physics, vol. 109, no. 19, pp. 8218-8224, 1998.

[33] T. Yanai, D. P. Tew, and N. C. Handy, "A new hybrid exchangecorrelation functional using the Coulomb-Attenuating Method (CAM-B3LYP)," Chemical Physics Letters, vol. 393, no. 1-3, pp. 51-57, 2004.

[34] M. J. Frisch, G. W. Trucks, H. B. Schlegel et al., Gaussian 09, Revision A.02, Gaussian Inc, Wallingford, Conn, USA, 2009.

[35] G. Olbrechts, T. Munters, K. Clays, A. Persoons, O.-K. Kim, and L.-S. Choi, "High-frequency demodulation of multi-photon fluorescence in hyper-Rayleigh scattering," Optical Materials, vol. 12, no. 2, pp. 221-224, 1999.

[36] G. Olbrechts, R. Strobbe, K. Clays, and A. Persoons, "Highfrequency demodulation of multi-photon fluorescence in hyper-Rayleigh scattering," Review of Scientific Instruments, vol. 69, no. 6, pp. 2233-2241, 1998.

[37] D. Rehm and A. Weller, "Kinetics of fluorescence quenching by electron and H-atom transfer," Israel Journal of Chemistry, vol. 8, no. 2, pp. 259-271, 1970.

[38] C. Y. Qin and A. E. Clark, "DFT characterization of the optical and redox properties of natural pigments relevant to dyesensitized solar cells," Chemical Physics Letters, vol. 438, no. 1-3, pp. 26-30, 2007.

[39] M. Grätzel and K. Kalyanasundaram, "Applications of functionalized transition metal complexes in photonic and optoelectronic devices," Coordination Chemistry Reviews, vol. 177, no. 1, pp. 347-414, 1998. 

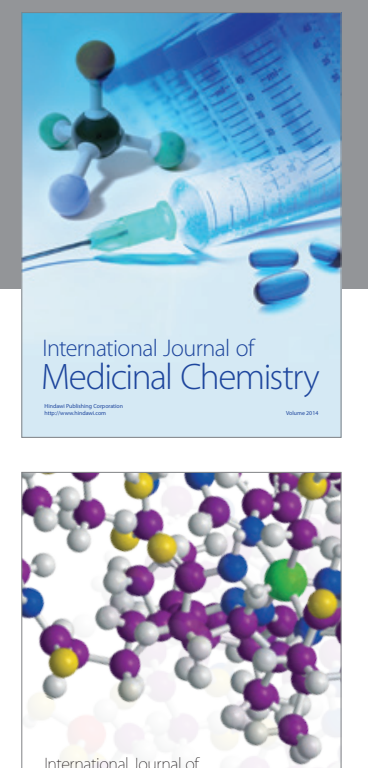

\section{Carbohydrate} Chemistry

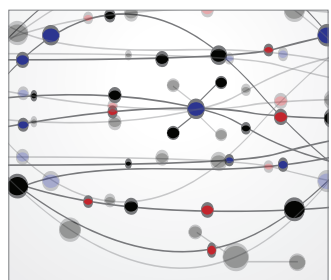

The Scientific World Journal
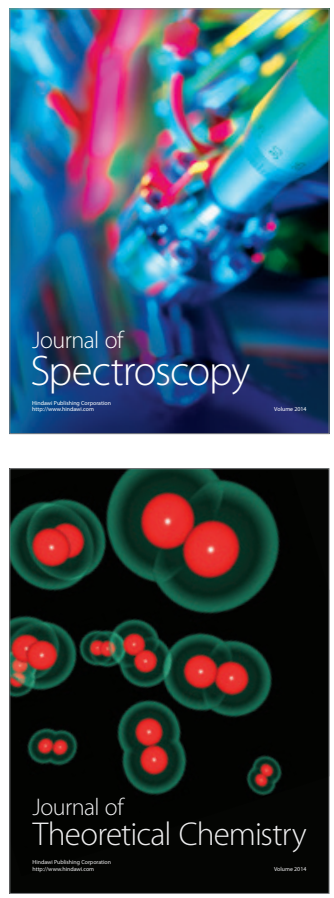
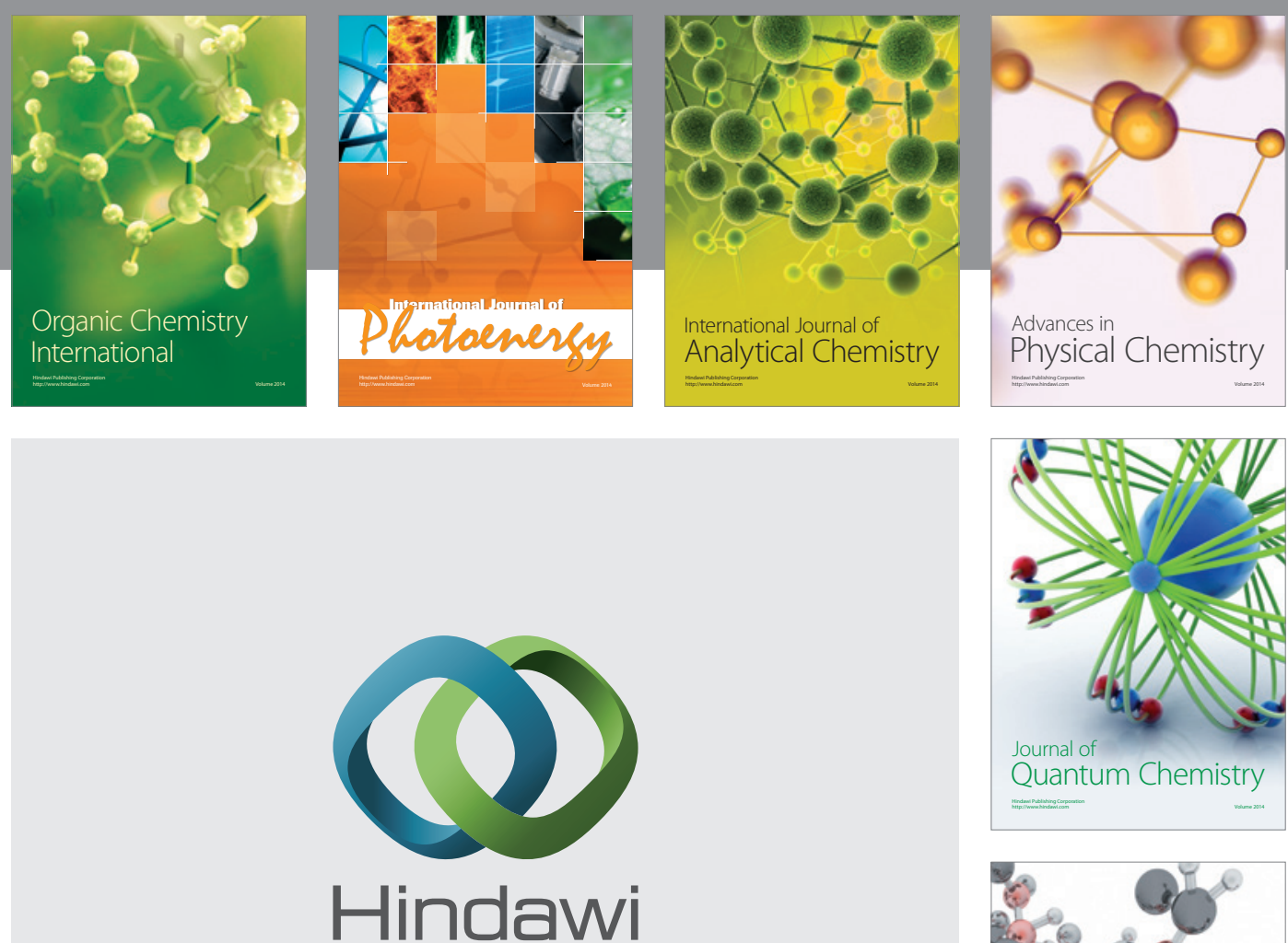

Submit your manuscripts at

http://www.hindawi.com

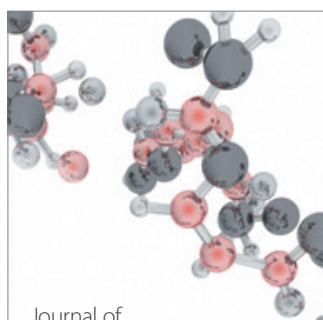

Analytical Methods

in Chemistry

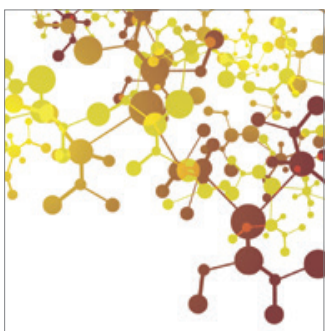

Journal of

Applied Chemistry

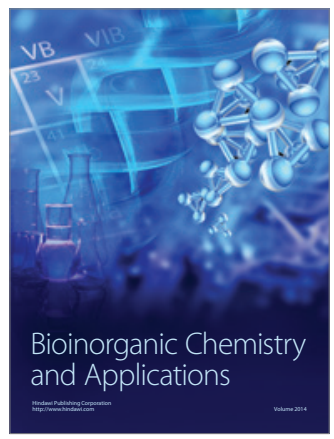

Inorganic Chemistry
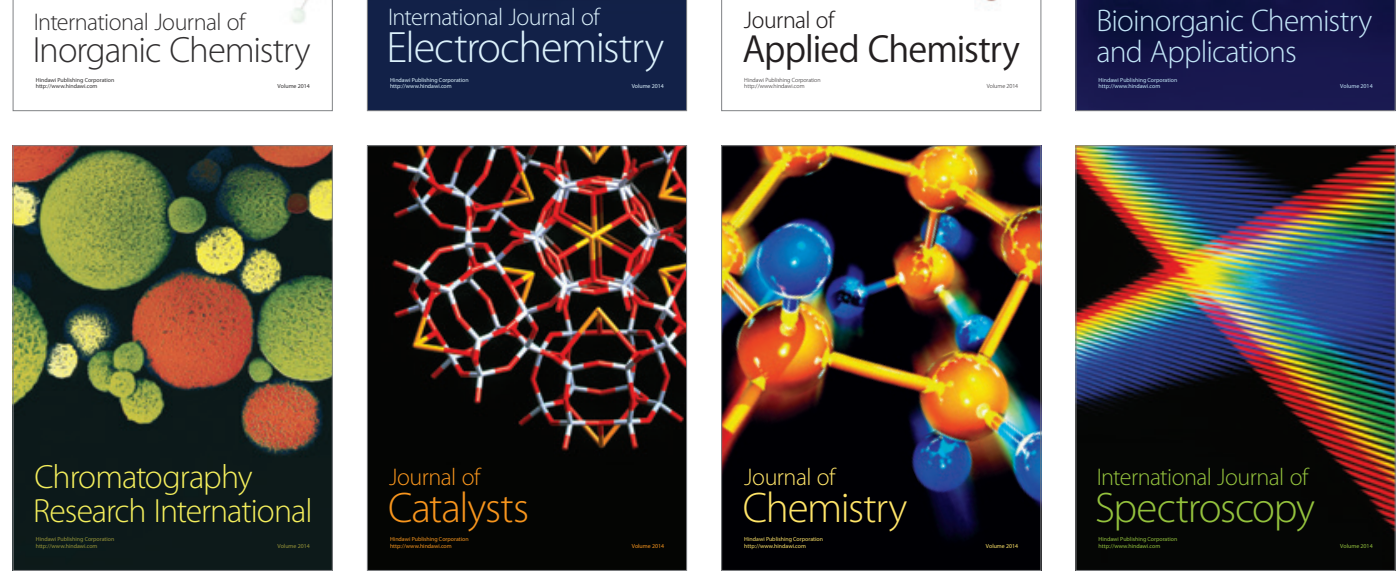